\title{
Améliorer le sentiment de compétence en gestion de projet des étudiants : résultats d'une démarche SoTL
}

Najoua Mohib, Simon Zingaretti and Rémi Bachelet

\section{(2) OpenEdition \\ Journals}

Electronic version

URL: https://journals.openedition.org/ripes/3399

DOI: 10.4000/ripes.3399

ISSN: 2076-8427

Publisher

Association internationale de pédagogie universitaire

\section{Electronic reference}

Najoua Mohib, Simon Zingaretti and Rémi Bachelet, "Améliorer le sentiment de compétence en gestion de projet des étudiants : résultats d'une démarche SoTL", Revue internationale de pédagogie de l'enseignement supérieur [Online], 37(3) | 2021, Online since 08 November 2021, connection on 27 September 2022. URL: http://journals.openedition.org/ripes/3399 ; DOI: https://doi.org/10.4000/ripes. 3399

This text was automatically generated on 27 September 2022.

\section{(c) (i) (ㅇ)}

Creative Commons - Attribution-NonCommercial-ShareAlike 4.0 International - CC BY-NC-SA 4.0 https://creativecommons.org/licenses/by-nc-sa/4.0/ 


\title{
Améliorer le sentiment de compétence en gestion de projet des étudiants : résultats d'une démarche SoTL
}

\author{
Najoua Mohib, Simon Zingaretti and Rémi Bachelet
}

\section{Introduction}

1 La qualité de l'enseignement supérieur représente, à l'heure actuelle, un enjeu majeur pour les décideurs politiques en Europe et, plus généralement, dans l'ensemble des pays du monde (De Ketele et al., 2016). Elle est considérée comme une condition nécessaire au développement d'une société qualifiée d'«innovante» et de «compétitive» permettant de mieux répondre aux mutations de la société et du monde du travail en particulier (Lanarès et Poteaux, 2013; Gerard et al., 2018). Longtemps promu par les instances politiques internationales telles que l'OCDE ou encore l'Union Européenne, ce type de discours est aujourd'hui relayé par les établissements d'enseignement supérieur (Granier et al., 2009). Une brève analyse de quelques documents stratégiques mis en ligne par des universités canadiennes, américaines, européennes ou encore asiatiques $^{1}$ montre ainsi que la qualité des enseignements constitue un objectif prioritaire pour soutenir la réussite des étudiants. Ce constat rejoint celui d'autres chercheurs (Rege Colet et Romainville, 2006; Langevin, 2007) qui expliquent que la qualité de l'enseignement constitue désormais un enjeu politique. C'est pour cette raison que des actions de formation à la pédagogie de l'enseignement supérieur se sont développées, au cours de ces dernières années, à l'initiative des directions d'établissements. Dans l'ensemble, les démarches de formation mises en place sont centrées sur le développement de l'expertise pédagogique et se réfèrent au modèle du «praticien réflexif» (Schön, 1983). Par ces démarches, il s'agit d'amener les enseignants à poser un regard critique sur leur activité professionnelle à partir d'une analyse de leurs pratiques d'enseignement et de leurs conséquences sur l'apprentissage 
des étudiants. Ce travail de réflexion et de distanciation peut être réalisé dans une perspective Scholarship of Teaching and Learning (SoTL) qui vise la production et le partage de connaissances pragmatiques en éducation (Boyer, 1990; Bernstein, 2010).

Dans cette perspective, cette contribution rend compte d'une action pédagogique qui s'inscrit dans un mouvement de "rénovation pédagogique " (Rege Colet et Romainville, 2006) en cours dans les universités françaises ainsi que des résultats d'une observation menée selon la démarche SoTL, en vue d'analyser les incidences d'une pratique d'enseignement de gestion de projet sur le sentiment de compétence des étudiants. Si le SoTL représente un courant de recherche à part entière (Endrizzi, 2011), peu d'études, conduites selon cette approche, sont diffusées dans l'espace scientifique francophone. Les publications en langue française liées au SoTL (Langevin et al., 2008; Endrizzi, 2011; Rege Colet et al., 2011; Lison, 2013; Bédard, 2014; Biémar et al., 2015; Rege Colet et Fanghanel, 2015) sont plutôt centrées sur l'histoire, la présentation, l'intérêt ou encore les limites de cette démarche. En s'inscrivant dans une logique SoTL, il s'agit de contribuer à la diffusion des connaissances en enseignement et apprentissage dans une perspective d'amélioration des pratiques pédagogiques tout en interrogeant l'effet "transformateur" d'une telle démarche sur le développement professionnel des enseignants-chercheurs qui la mettent en œuvre à l'université.

Pour ce faire, une première partie présente le contexte pédagogique ainsi que le cadre théorique qui étaye en partie la conception du dispositif pédagogique expérimenté. Les modalités de collecte et d'analyse des données sont ensuite décrites avant l'explicitation des résultats des observations. Une discussion synthétisant les principaux enseignements de cette recherche vient clore l'article, donnant l'occasion de revenir sur cette expérience d'exploitation d'une démarche SoTL particulière.

\section{Contexte institutionnel et théorique}

4 Cette partie présente une description du contexte d'enseignement permettant, d'une part, d'illustrer la démarche SoTL proposée par Bélisle et al. (2016), qui a été adoptée ici, et, d'autre part, de montrer le passage d'une réflexion pédagogique à la formulation d'une problématique de recherche.

\subsection{Présentation du dispositif pédagogique}

5 L'observation porte sur un dispositif pédagogique intitulé Mooc-PIC qui a obtenu un soutien financier de l'Université de Strasbourg dans le cadre d'un appel à projets Initiatives d'Excellence (IdEx) visant à soutenir les pratiques de formations jugées innovantes en licence et en master. Ce dispositif repose sur une approche par compétence (Boutin, 2004) et a pour objectif de contribuer à la professionnalisation des étudiants inscrits en master de sciences de l'éducation et de la formation (SEF). Concrètement, il vise à favoriser chez eux le développement des compétences en gestion de projet considérées aujourd'hui comme essentielles dans les métiers de l'ingénierie et de l'intervention en formation (ex. conseiller pédagogique, ingénieur pédagogique, responsable de formation, etc.). La notion de « compétence » renvoie ici aux travaux de Roegiers (2014, p. 73) qui indique qu'il existe désormais un consensus en SEF pour la définir comme « une mobilisation intériorisée et réfléchie d'un ensemble de ressources pour faire face à des situations complexes ». Ainsi, le dispositif Mooc-PIC 
s'étale sur une année universitaire et intègre deux modules semestriels complémentaires qui accordent une importance particulière aux activités de mise en pratique et aux retours constructifs grâce à une structuration pédagogique spécifique (voir Tableau 1).

Tableau . Présentation du dispositif pédagogique Mooc-PIC

\begin{tabular}{|l|l|l|}
\hline Module & Mooc & PIC \\
\hline Objectifs & $\begin{array}{l}\text { Initiation à la gestion de } \\
\text { projet }\end{array}$ & $\begin{array}{l}\text { Maîtrise de la gestion } \\
\text { de projet }\end{array}$ \\
\hline Démarche & $\begin{array}{l}\text { xMooc, pédagogie } \\
\text { transmissive }\end{array}$ & $\begin{array}{l}\text { Mise en situation réelle } \\
\text { pédagogie par projet }\end{array}$ \\
\hline Outil & Plateforme numérique & Plateforme collaborative \\
\hline Accompagnement & Entraide étudiante & Tutorat assuré par un expert \\
\hline Évaluation & $\begin{array}{l}\text { Évaluation par les pairs } \\
\text { Quizz et devoirs (1 fois par } \\
\text { semaine) }\end{array}$ & $\begin{array}{l}\text { Auto-, co- et hétéro-évaluation (pairs, jury, } \\
\text { Livrables et soutenance orale } \\
\text { à mi-parcours }\end{array}$ \\
\hline $\begin{array}{l}\text { Espace } \\
\text { d'apprentissage }\end{array}$ & Hors université & Université + Hors Université \\
\hline
\end{tabular}

Le premier module, intitulé Mooc Gestion de projet est proposé à distance dès le début du premier semestre. Produit par une équipe de bénévoles, de startups (Unow, LearnGdP, MOOCit) et Centrale Lille, ce Mooc a été intégré dans la formation des étudiants afin de les initier à la gestion de projet par la découverte des notions fondamentales du management et des outils de conception et de pilotage (par exemple: préparer une étude d'investissement, établir un planning, faire la synthèse d'une réunion, négocier un objectif). Le deuxième module, dénommé PIC (Projet, Innovation, Conception) est organisé en présentiel au second semestre. Il succède au Mooc et vise la maîtrise de la conception et du pilotage de projet à partir d'une mise en situation professionnelle. Les étudiants développent un projet innovant en éducation et/ou en formation pour le compte d'un commanditaire externe (institution, entreprise, association, etc.). L'ensemble Mooc-PIC repose donc sur une articulation «distance-présence » et «théorie-pratique » censée favoriser la progression des apprentissages en permettant aux étudiants : 1) d'exploiter les notions de gestion de projet qu'ils ont étudiées; 2) de bénéficier des retours constructifs par le système d'évaluation et des modalités d'accompagnement mis en place.

\subsection{Une démarche ancrée sur la pratique pédagogique}

7 La conception du dispostif Mooc-PIC a été motivée par les constats réalisés à l'issue de la première année d'enseignement, organisé sous la forme d'un travail dirigé (TD), visant à faire découvrir la gestion de projet par des études de cas et d'une mise en situation réelle. Au moment de l'évaluation de cet enseignement, les étudiants avaient exprimé un faible sentiment de compétence en gestion de projet, la plupart d'entre eux ayant déclaré ne pas se sentir « prêts » à conduire un « vrai » projet. Cette situation avait alors 
conduit à solliciter les services d'un conseiller pédagogique pour réfléchir aux moyens d'améliorer les pratiques pédagogiques. C'est dans le cadre d'un accompagnement individualisé, assuré par le centre de soutien à l'enseignement et à l'apprentissage de l'université que cet enseignement a été questionné selon une démarche SoTL (Bélisle et al., 2016) déclinée en six étapes itératives (Bélisle et al., 2016), suivies de manière non linéaire (voir Tableau 2).

Tableau 2. Démarche SoTL (Bélisle et al., 2016) appliquée au dispositif Mooc-PIC

\begin{tabular}{|l|l|}
\hline Démarche SoTL & Exploitation dans le cas du Mooc-PIC \\
\hline 1. Analyse de la pratique & $\begin{array}{l}\text { Formulation du questionnement pédagogique : Comment améliorer le } \\
\text { sentiment de compétence en gestion de projet des étudiants? }\end{array}$ \\
\hline $\begin{array}{l}\text { 2. Appropriation des } \\
\text { connaissances }\end{array}$ & $\begin{array}{l}\text { Réalisation d'un état de l'art sur la notion de « sentiment de } \\
\text { compétence » et définition du cadre théorique }\end{array}$ \\
\hline $\begin{array}{l}\text { 3. Conception du } \\
\text { changement }\end{array}$ & Réingénierie de la formation \\
\hline $\begin{array}{l}\text { 4. Implantation du } \\
\text { changement }\end{array}$ & Planification des activités d'apprentissage \\
\hline $\begin{array}{l}\text { 5. Évaluation } \quad \mathrm{du} \\
\text { changement }\end{array}$ & $\begin{array}{l}\text { Mise en place d'un protocole de recherche et d'une évaluation de } \\
\text { l'enseignement par les étudiants }\end{array}$ \\
\hline $\begin{array}{l}\text { 6. Communication } \\
\text { changement }\end{array}$ & Publication scientifique \\
\hline
\end{tabular}

8 La première étape (analyse de pratique) a permis de formuler le problème pédagogique de manière explicite : comment favoriser le sentiment de compétence en gestion de projet chez les étudiants et améliorer ainsi leur expérience d'apprentissage? Au cours de la deuxième étape (appropriation des connaissances), un état de l'art a été réalisé sur la notion de "sentiment de compétence " dans le but de problématiser le questionnement initial (voir section suivante). La troisième étape (conception du changement) a conduit à reprendre l'enseignement en réfléchissant aux moyens qui permettraient aux étudiants de développer des compétences en gestion de projet afin qu'ils se sentent capables de concevoir et de diriger un projet. Le passage en revue de la littérature scientifique et la réalisation d'une veille pédagogique ont débouché sur:1) l'introduction du Mooc Gestion de projet, fondé sur l'évaluation par les pairs; 2) la mise en place du module PIC reposant sur le tutorat par un expert contribuant à encourager et à soutenir les étudiants durant la phase de mise en situation de projet. Cette étape de réingénierie pédagogique a aussi abouti à l'élaboration d'un référentiel de compétences en gestion de projet comprenant 24 micro-compétences, définies en termes de tâches professionnelles et regroupées selon trois domaines d'activités : la gestion managériale; la gestion technique; la gestion des moyens. Une telle catégorisation a été inspirée des travaux de l'association francophone de management de projet (AFITEP, 2000). La quatrième étape (implantation du changement) a consisté à planifier et à mettre en œuvre le déroulement des deux modules de formation (Mooc et PIC) tout en les articulant. Dans la cinquième étape (évaluation du changement), un protocole de recherche (décrit dans la partie méthodologie) a permis de procéder au bilan de cet enseignement afin de recueillir la perception des étudiants sur les éventuels apports 
et/ou limites de l'environnement Mooc-PIC. La publication de cet article fait partie de la sixième étape (communication du changement) en vue de contribuer à la formalisation de connaissances en pédagogie de l'enseignement supérieur. L'ensemble de ces étapes relève d'un processus de collaboration entre des enseignants-chercheurs et des conseillers pédagogiques, orienté vers « l'amélioration de l'apprentissage des étudiants et des pratiques enseignantes » (Bélisle et al., 2016, p. 77).

\subsection{Problématique de recherche}

9 La revue de littérature a permis de constater que de nombreux travaux en SEF soulignent le rôle déterminant du sentiment de compétence (ou d'efficacité) dans l'apprentissage (Cosnefroy, 2010; Berger et Büchel, 2012; Peguret, 2014), notamment lors d'un apprentissage autorégulé (Noël et Cartier, 2016; Jacob, 2018). Celui-ci joue un rôle essentiel dans la réussite académique des étudiants car il favorise l'utilisation de stratégies d'apprentissage performantes (Lecomte, 2004). D'après Zimmerman (1995, 2000), il constitue l'un des principaux déterminants de la motivation en agissant à la fois sur le choix des tâches à accomplir, l'engagement, l'effort, la persistance ou encore la gestion des émotions. Le sentiment de compétence (ou d'efficacité) stimule, selon ce même auteur, les conduites d'autorégulation, telles que la vérification, l'autoévaluation, la fixation de buts à atteindre, permettant aux apprenants de prendre le contrôle de leur apprentissage. Il a été montré, par ailleurs, que pour atteindre une performance, la confiance du sujet en ses capacités d'apprentissage est tout aussi déterminante que la compétence réelle (Galand, 2011).

Ainsi, le modèle de l'auto-efficacité (Bandura, 2003) a paru pertinent pour appréhender ce qui pourrait contribuer au développement du sentiment de compétence en gestion de projet chez les étudiants participant à la formation. Cette théorie sociocognitive établit que les individus sont des « agents proactifs » qui peuvent exercer une influence sur leur comportement et que leurs croyances d'efficacité jouent un rôle central dans le contrôle de l'action et la régulation cognitive de la motivation. Autrement dit, «plus l'efficacité personnelle perçue est forte, plus les buts que les personnes se fixent sont élevés et plus leur engagement est solide» (Bandura, 2003, p. 181). L'efficacité personnelle perçue (self-efficacy) renvoie ainsi aux «évaluations par l'individu de ses aptitudes personnelles " (Bandura, 2003, p. 24). Si la notion se distingue de celle d'«estime de soi », qui fait plutôt référence aux "évaluations de sa valeur personnelle », elle a tendance à se confondre avec le « sentiment de compétence » bien que des nuances aient été repérées (Carré, 2004; Galand et Vanlede, 2004; Cosnefroy, 2007; Carré, 2010). La principale différence entre les deux termes tiendrait au fait que la compétence perçue désigne une perception globale de ses capacités d'action tandis que le sentiment d'efficacité porterait sur des activités spécifiques (Carré, 2004). Un des résultats les plus diffusés de la théorie de Bandura (2003) concerne la construction des jugements d'auto-efficacité qui puiserait à quatre sources d'information: l'expérience active de maîtrise (tout ce qui peut servir, dans son passé, d'indicateurs de capacité), l'expérience vicariante (comparaison avec ce que font les autres pour évaluer ses propres compétences), la persuasion verbale (messages adressés, par des personnes jugées crédibles, à propos de ses capacités) et les états physiologiques et émotionnels (informations transmises par les réactions somatiques). Ces résultats ont conduit à accorder, dans le cas de cette expérience pédagogique, une plus grande place aux activités de mise en pratique de gestion de projet déjà proposées dans l'enseignement 
initial et à concevoir des modalités pédagogiques ayant recours à la persuasion verbale. Grâce à un ensemble de modalités d'accompagnement (tutorat, entraide étudiante) et d'évaluation (évaluation par les pairs, co/hétéro-évaluation, évaluation critériée et continue) qui ont toutes été explicitées, il s'est agit d'aider les étudiants à progresser tout au long de leur parcours en leur adressant, de manière formelle et informelle, soutien, encouragements, conseils et retours constructifs.

11 La question de recherche suivante a ainsi guidé le travail réalisé dans une perspective SoTL: dans quelle mesure des modalités pédagogiques offrant aux étudiants des situations de persuasion verbale d'une part et des opportunités d'expériences actives de maitrise d'autre part favorisent-elles le sentiment de compétence en gestion de projet? L'hypothèse avancée était que les modalités pédagogiques ayant recours à la persuasion verbale ont une incidence positive plus marquée sur le sentiment de compétence en gestion de projet des participants que celles qui relèvent de l'expérience active de maîtrise.

\section{Méthodologie}

12 Une démarche méthodologique fondée sur une approche mixte a été utilisée pour répondre à notre question de recherche. Premièrement, une enquête par questionnaires a été proposée aux étudiants participant au dispositif Mooc-Pic dans le but de mesurer l'évolution de leur sentiment de compétence en gestion de projet (approche quantitative). Deuxièmement, des entretiens semi-directifs ont été menés auprès de ces mêmes étudiants afin d'approfondir et de mieux comprendre les résultats des questionnaires (approche qualitative). Au total, dix étudiants (E1 à E10), inscrits en deuxième année de master SEF, ont volontairement participé à cette recherche.

\subsection{Approche quantitative}

13 En premier lieu, les étudiants ont complété un questionnaire ${ }^{2}$ présenté sous la forme d'une grille d'auto-évaluation reprenant les 24 items du référentiel de compétence élaboré au démarrage de ce travail. Il a semblé plus pertinent de construire un instrument de mesure spécifique visant à repérer les incidences de la formation. Pour chacune des tâches énoncées, les étudiants devaient indiquer le degré de maitrise ressenti sur une échelle de Likert à quatre niveaux ${ }^{3}$. Trois passations ont été organisées à des moments jugés clés par l'équipe pédagogique : avant le démarrage du Mooc (T1); après la validation du Mooc (T2); puis à l'issue de la soutenance orale PIC (T3). Il s'agissait alors d'observer l'évolution du sentiment de compétence en gestion de projet exprimé par les étudiants avant, pendant puis à la fin de leur formation. Le questionnaire a ainsi permis d'établir une échelle globale du sentiment de compétence, subdivisé en trois sous échelles: "gestion managériale »; "gestion technique»; " gestion des moyens".

14 Au total, 22 items sur 24 ont été retenus (2 items auxquels quelques participants n'avaient pas répondu ont été supprimés). La fiabilité du questionnaire a été vérifiée au moyen de l'alpha de Cronbach. 
Tableau 3. Moyennes, écart-types et scores de fiabilité aux trois passations

\begin{tabular}{|c|c|c|c|c|c|c|c|c|c|}
\hline & \multicolumn{3}{|l|}{ T1 } & \multicolumn{3}{|l|}{ T2 } & \multicolumn{3}{|l|}{ T3 } \\
\hline & Moy. & E-T & $\alpha$ Cronbach & Moy. & E-T & $\alpha$ Cronbach & Moy. & E-T & $\alpha$ Cronbach \\
\hline Échelle globale & 49.18 & 7.63 & .786 & 57.91 & 6.99 & .880 & 62.73 & 7.06 & .909 \\
\hline Gestion managériale & 20.45 & 2.62 & .704 & 23.82 & 3.74 & .922 & 24.09 & 3.18 & .881 \\
\hline Gestion technique & 14.82 & 4.17 & $.623^{*}$ & 17.55 & 2.54 & .704 & 19.82 & 2.23 & $.607^{*}$ \\
\hline Gestion des moyens & 13.91 & 3.02 & $.672^{*}$ & 16.55 & 2.34 & .702 & 18.82 & 2.27 & .787 \\
\hline
\end{tabular}

La consistance interne pour l'échelle globale est satisfaisante (voir Tableau 3) à T1, T2 et T3 $(.78<\alpha<.90)$. En ce qui concerne les trois sous-échelles, les coefficients varient entre .607 et .922 . Toutes les variables ont été conservées car la qualité ne s'améliore pas lorsque l'un ou l'autre item est retiré. Par ailleurs, les trois valeurs inférieures à .7 $(\alpha=, 607 ; \alpha=, 623 ; \alpha=, 672)$ restent acceptables.

Une série de tests de Wilcoxon unilatéraux ${ }^{4}$ a été réalisée pour juger de l'éventuelle significativité des différences entre les passations (T1; T2; T3) et les dimensions de l'échelle (gestion managériale; gestion technique; gestion des moyens).

\subsection{Approche qualitative}

17 En second lieu, des entretiens semi-directifs, d'une durée moyenne de 45 minutes, ont été menés avec chaque étudiant. Il s'agissait de recueillir leurs perceptions des résultats aux questionnaires et de repérer les conséquences directes des modalités pédagogiques ayant recours à la persuasion verbale et de celles offrant des opportunités d'expériences actives de maîtrise sur le sentiment de compétence en gestion de projet. Par conséquent, les réponses fournies par les étudiants aux différents questionnaires leur ont été présentées durant l'entretien sous la forme d'un radar (voir Figure 1) leur permettant d'analyser plus facilement l'évolution de leur sentiment de compétence entre les trois temps de la passation (T1, T2 et T3). 
Figure 1. Exemple d'une représentation graphique en radar des réponses fournies par un étudiant

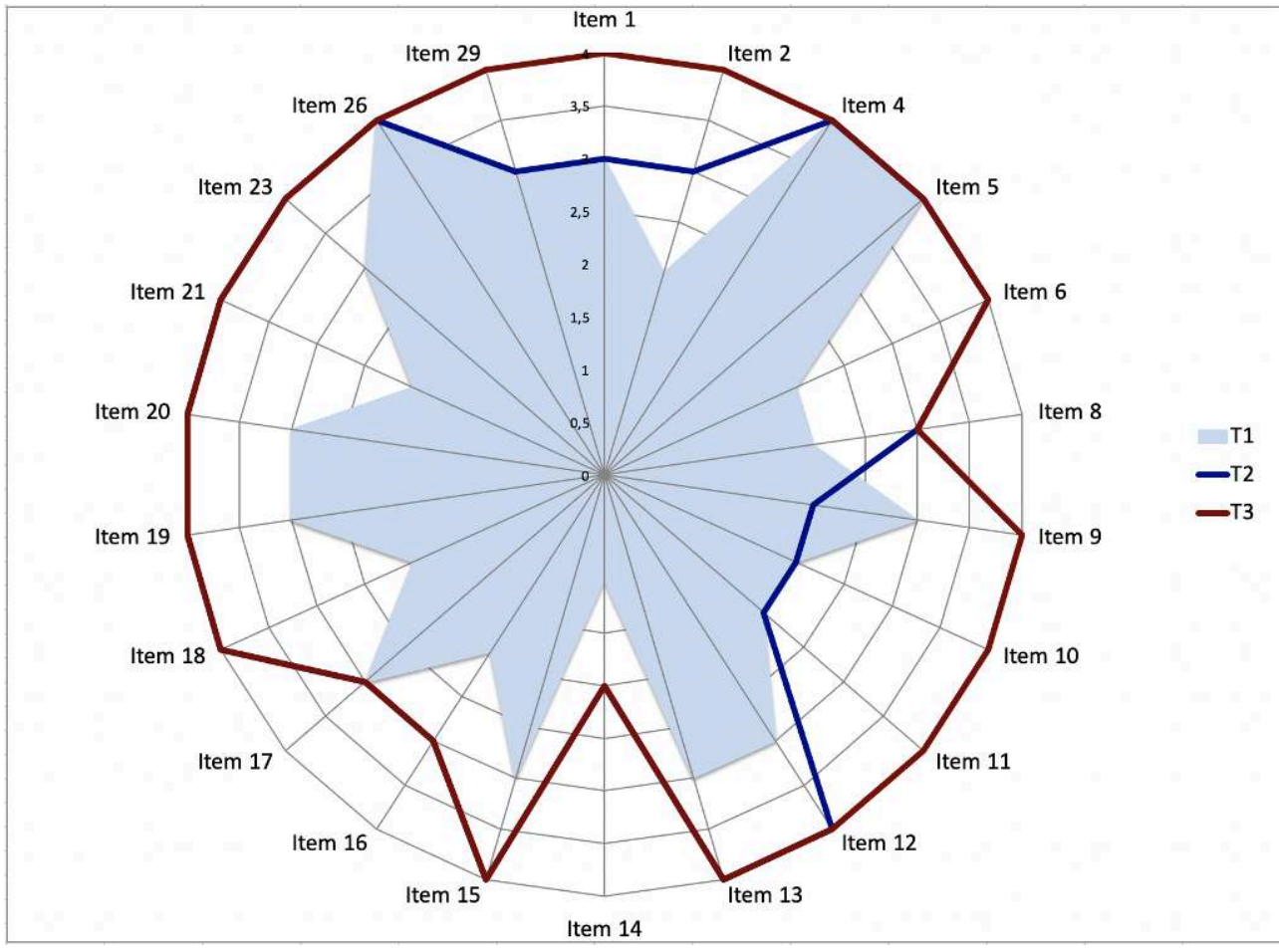

18 Par ailleurs, les entrevues ont été conduites à l'aide d'un guide d'entretien composé de quatre thèmes détaillés dans le tableau 4 ci-dessous :

Tableau 4. Guide d'entretien

\begin{tabular}{|l|l|}
\hline Thème & Questions \\
\hline Vécu du dispositif & $\begin{array}{l}\text { Quel regard portez-vous sur le dispositif Mooc-PIC? Que pensez-vous de son } \\
\text { organisation pédagogique? Quel est selon vous son intérêt principal? }\end{array}$ \\
\hline $\begin{array}{l}\text { Influence } \\
\text { sentiment } \\
\text { compétence } \quad \mathbf{d u} \\
\text { gestion de projet }\end{array}$ & $\begin{array}{l}\text { Est-ce que vous aviez une expérience de gestion de projet avant ce master? } \\
\text { Comment vous situez-vous, dans le domaine de la gestion de projet, par } \\
\text { rapport à d'autres personnes, notamment vos collègues de la promotion? } \\
\text { Est-ce que vous avez eu des retours sur votre travail dans le cadre de votre } \\
\text { participation à ce dispositif? Lesquels? Si oui, comment vous en êtes-vous } \\
\text { servis? Si non, cela vous a-t-il manqué? }\end{array}$ \\
\hline $\begin{array}{l}\text { Évolution } \\
\text { sentiment } \\
\text { compétence } \\
\text { gestion de projet }\end{array}$ & $\begin{array}{l}\text { Que comprenez-vous de ce radar? Qu'évoque-t-il pour vous? Comment vous } \\
\text { entes-vous évalués? Comment vous êtes-vous attribué les scores? Est-ce que } \\
\text { vous avez procédé de la même manière à chaque fois (T1, T2, T3)? Comment } \\
\text { expliquez-vous cette évolution? (illustration avec un item qui montre une } \\
\text { progression entre T1 et T2 puis une stabilisation ou régression entre T2 et } \\
\text { T3). }\end{array}$ \\
\hline $\begin{array}{l}\text { Question ouverte } \\
\text { pour terminer } \\
\text { l'entretien }\end{array}$ & $\begin{array}{l}\text { Est-ce qu'il y a une question, que nous n'avons pas posée, et à laquelle vous } \\
\text { auriez aimé répondre? }\end{array}$ \\
\hline
\end{tabular}

19 Les entretiens ont tous été enregistrés avec l'accord des participants puis retranscrits dans leur intégralité avant de faire l'objet d'une analyse de contenu thématique 
(Muchielli, 2006). Pour procéder au codage des unités de sens (mots, expressions ou phrases liés à une même idée), une grille d'analyse a été construite à partir du cadre conceptuel de cette étude (Bandura, 2003). Cependant, seules trois des quatre sources du sentiment d'auto-efficacité ont été retenues pour déterminer les catégories de la grille d'analyse à savoir : l'« expérience active de maitrise ", l'« expérience vicariante " et la "persuasion verbale». La source "états physiologiques et émotionnels", considérée comme pertinente, principalement dans le domaine du sport ou de la santé (Bandura, 2003), n'a pas été retenue compte tenu de l'implication des chercheurs dans la formation universitaire des participants qui auraient pu rencontrer des difficultés à exprimer leurs affects. Chacune des trois catégories a ainsi été subdivisée en plusieurs sous-catégories, elles-mêmes associées à des descripteurs qui ont été définis, au départ, à l'aide des descriptions proposés par d'autres chercheurs qui ont étudié le sentiment d'auto-efficacité (Galand et Vanlede, 2004; Melançon et al., 2013; Guy et Hubert, 2014). Toutefois, quelques ajustements des sous-catégories et des descripteurs préalablement définis se sont révélés nécessaires durant le codage.

Afin de garantir la fiabilité du travail d'analyse et de valider la grille présentée ici (voir Tableau 5), la technique d'inter-codage proposée par Miles et Huberman (2003) a été utilisée. Il s'agit d'un procédé consistant à réaliser, de manière simultanée, un codage des données par au moins deux chercheurs afin de calculer le degré d'accord inter-juge selon la formule suivante: nombre d'accords divisé par le nombre d'accords plus le nombre de désaccords $[A /(A+D)]$. Concrètement, un double codage de l'ensemble des entretiens $(n=10)$ a été réalisé par l'enseignant-chercheur concepteur du dispositif Mooc-Pic et le conseiller pédagogique engagé dans ce projet, et ce à deux reprises. Un degré d'accord inter-juge de $75 \%$ a pu être établi à l'issue de la première phase d'intercodage qui a permis de discuter des points de divergence et d'ajuster certains éléments de la grille d'analyse notamment les sous-catégories et les descripteurs. Lorsque la deuxième phase d'inter-codage s'est terminée, il a été possible de constater un degré d'accord inter juge supérieur à celui obtenu lors du premier codage, soit un taux de $89,5 \%$, ce qui est considéré comme très satisfaisant selon Miles et Huberman (2003).

Tableau 5. Grille d'analyse

\begin{tabular}{|c|c|c|c|}
\hline Catégorie & Code & Sous-catégorie & Descripteurs \\
\hline \multirow{3}{*}{$\begin{array}{l}\text { Expérience } \\
\text { active de } \\
\text { maîtrise }\end{array}$} & \multirow{3}{*}{ XPM } & Auto-observation & $\begin{array}{l}\text { Histoire, parcours de formation, souvenirs, expérience, } \\
\text { succès ou échec vécus }\end{array}$ \\
\hline & & Effort fourni & Implication, difficulté, facilité \\
\hline & & $\begin{array}{l}\text { Facteurs } \\
\text { contextuels }\end{array}$ & $\begin{array}{l}\text { Circonstances dans lesquelles l'activité est réalisée, } \\
\text { ressources (matérielles, humaines) disponibles, } \\
\text { obstacles situationnels }\end{array}$ \\
\hline \multirow[b]{2}{*}{$\begin{array}{l}\text { Expérience } \\
\text { vicariante }\end{array}$} & \multirow[b]{2}{*}{ XPV } & Modelage & $\begin{array}{l}\text { Observation du comportement d'autrui, valeur } \\
\text { accordée au modèle, identification, inspiration }\end{array}$ \\
\hline & & Comparaison & $\begin{array}{l}\text { Similitude ou différence de caractéristique personnelle } \\
\text { (ex. âge sexe, niveau éducatif, ..), similitude ou } \\
\text { différence de performance (ex. résultats aptitude), } \\
\text { comparaison égale, supérieure, inférieure }\end{array}$ \\
\hline
\end{tabular}




\begin{tabular}{|l|l|l|l|}
\hline & & $\begin{array}{l}\text { Feedback sur les } \\
\text { aptitudes }\end{array}$ & Appréciation des capacités, du potentiel \\
\cline { 2 - 4 } $\begin{array}{l}\text { Persuasion } \\
\text { verbale }\end{array}$ & PV & $\begin{array}{l}\text { Feedback sur les } \\
\text { performances }\end{array}$ & Appréciation des résultats (ex. notes, livrables, ...) \\
\cline { 2 - 4 } & $\begin{array}{l}\text { Messages } \\
\text { adressés }\end{array}$ & $\begin{array}{l}\text { Commentaires, conseils, encouragements, avis, } \\
\text { soutiens, appréciations }\end{array}$ \\
\hline
\end{tabular}

\section{Résultats}

21 Cette section résume les principaux résultats des volets quantitatif et qualitatif de l'étude : 1) le constat de l'évolution du sentiment de compétence en gestion de projet des étudiants tout au long de la formation; 2) le repérage des sources d'information évoquées par les étudiants permettant notamment de comprendre les résultats obtenus aux questionnaires.

\subsection{Augmentation du sentiment de compétence en gestion de projet}

22 L'analyse des scores (voir Tableau 3) montre que le sentiment de compétence en gestion de projet (échelle globale) augmente au cours du temps (moyenne T1 =49,18; moyenne $\mathrm{T} 2=57,91 ;$ moyenne $\mathrm{T} 3=62,73$ ) et ce, de manière significative et entre chaque mesure (tests de Wilcoxon: $\mathrm{T}_{\mathrm{T} 1-\mathrm{T} 3}=0.0 ; \mathrm{p}<.01 ; \mathrm{T}_{\mathrm{T} 1-\mathrm{T} 2}=9,0 ; \mathrm{p}<.05 ; \mathrm{T}_{\mathrm{T} 2-\mathrm{T} 3}=8 ; \mathrm{p}<.05$ ). Cette augmentation significative s'observe aussi pour chacune des sous-échelles ("gestion managériale ", " gestion technique ", " gestion des moyens ») et entre chaque mesure, à l'exception de la sous-échelle de gestion des moyens qui n'augmente plus entre $\mathrm{T} 2$ et $\mathrm{T} 3\left(\mathrm{~T}_{\mathrm{T} 2-\mathrm{T} 3}=15.0 ; \mathrm{ns}\right)$.

Si un tel constat est appréciable d'un point de vue pédagogique, comment les étudiants expliquent-ils l'évolution de leur sentiment de compétence en gestion de projet au regard des situations de persuasion verbale et des opportunités d'expériences actives de maîtrise offertes par le dispositif Mooc-PIC?

\subsection{Explications données par les étudiants}

En observant la fréquence des propos (voir Tableau 6) pour chacune des catégories relatives aux sources d'information du sentiment de compétence en gestion de projet, il apparaît que les étudiants évoquent le plus souvent l'expérience active de maitrise $(51,59 \%)$. La persuasion verbale $(32,98 \%)$ et l'expérience vicariante $(15,43 \%)$ sont également mentionnées mais dans une moindre proportion. Pour chacune de ces sources, les propos manifestant une influence positive sur le sentiment de compétence dominent.

Tableau 6. Fréquence des propos relatifs aux sources d'information du sentiment de compétence

\begin{tabular}{|l|l|l|l|l|}
\hline Catégorie & $\begin{array}{l}\text { Propos manifestant } \\
\text { une influence positive }\end{array}$ & $\begin{array}{l}\text { Propos manifestant } \\
\text { une influence négative }\end{array}$ & $\begin{array}{l}\text { Nbre total } \\
\text { de propos }\end{array}$ & $\begin{array}{l}\text { Part des } \\
\text { propos }\end{array}$ \\
\hline
\end{tabular}




\begin{tabular}{|l|l|l|l|l|}
\hline $\begin{array}{l}\text { Expérience active } \\
\text { de maîtrise }\end{array}$ & 76 & 21 & 97 & $51,59 \%$ \\
\hline $\begin{array}{l}\text { Persuasion } \\
\text { verbale }\end{array}$ & 43 & 19 & 62 & $32,98 \%$ \\
\hline $\begin{array}{l}\text { Expérience } \\
\text { vicariante }\end{array}$ & 23 & 6 & 29 & $15,43 \%$ \\
\hline Total des propos & 142 & 46 & 188 & $100 \%$ \\
\hline
\end{tabular}

Cette section présente les liens éventuels que font les étudiants entre les deux sources d'information principales (expérience active de maîtrise et persuasion verbale) et l'évolution de leur sentiment de compétence observé à l'issue de leur formation.

\subsubsection{Expérience active de maîtrise}

26 Tous les étudiants interrogés se réfèrent à leurs expériences réussies pour expliquer l'augmentation de leur sentiment de compétence en gestion de projet: «Là où j'ai vraiment expérimenté, il y a une progression et là ou je n'ai pas expérimenté et je n'ai pas mis en place mes compétences, il n'y a pas de progression » (E1).

Ainsi, les étudiants se sont référés, au moment de la première passation (T1), c'est-àdire avant leur participation au dispositif Mooc-PIC, à leurs réussites antérieures à la formation: "Je suis restée sur mon vécu antérieur, mes expériences antérieures. J'ai eu une bonne maîtrise " (E5); "Je faisais référence aux expériences antérieures pour le T1 (...) Cela faisait l'écho à des choses que j'avais déjà vues (...) Je savais faire. Je me sentais relativement à l'aise » (E9). En revanche, pour les passations suivantes (T2 et T3), c'est-à-dire à l'issue du Mooc puis du module PIC, tous mettent en évidence l'influence positive des succès vécus au cours de la formation: «Pour le deuxième, je me suis dit qu'après le Mooc, cela tu peux le faire, ceci tu sais le faire, tu as vu que tu peux le faire 》 (E8); « Au troisième moment, je faisais référence au PIC, vu que j'avais un exemple tout à fait clair » (E9). "Il y a un énorme progrès et maîtrise de l'item [rédiger un compte rendu de réunion], parce que j'ai réalisé l'entretien, la synthèse, la retranscription [pour le PIC] 》 (E5).

Certes, les étudiants, dans leur grande majorité, (8 sur 10) évoquent des difficultés rencontrées au cours de leur expérience PIC, qui ont d'ailleurs eu un effet négatif sur le jugement de l'une ou l'autre de leurs compétences: "Durant le PIC je me suis rendu compte que ce n'est pas forcément là où je suis le plus à l'aise, d'informer de l'état d'avancement, surtout aux commanditaires (...) Je pensais au début être à l'aise (...), finalement de par l'expérience du PIC, ce n'est pas là ou j'excelle le plus. Donc je n'ai pas pu mettre 4, j'ai simplement mis le 3 » (E7); "J'ai revu aussi à la baisse un certain nombre de compétences que je pensais avoir développé après le Mooc. Une fois dans le projet PIC je me suis rendu compte que je ne maîtrisais pas autant que ce que je pensais " (E8). Cependant, la plupart évoque une progression de leurs capacités et considèrent même que la mise en situation réelle, organisée dans le cadre du module PIC, a contribué à améliorer leurs compétences comme en témoignent ces trois étudiants : «Pour la plupart des compétences, je sentais que j'étais capable de faire des choses au fur et à mesure. Après la pratique, le PIC, j'étais certaine que j'étais capable de le faire " (E1); "L'item 4 [impliquer les membres de l'équipe] a augmenté suite à mon expérience en PIC » (E6). «À T3, il y a le PIC qui est passé par là où il y a eu une 
ébauche de cahier des charges qui a été fait. Cette pratique m'a conforté dans la capacité de pouvoir le faire » (E8).

Ainsi, les déclarations des étudiants indiquent qu'ils se sont principalement centrés sur les succès, voire les échecs vécus, pour juger s'ils étaient en mesure de maitriser telle ou telle autre tâche liée à la gestion de projet.

\subsubsection{Persuasion verbale}

30 La quasi-totalité des étudiants (8 sur 10) considère que les retours qu'ils ont reçus au cours de leur formation influencent positivement leurs croyances en leurs capacités à gérer un projet : «Cela aide à identifier ses progressions, à identifier son cheminement » (E5); "Nous avons eu beaucoup de retours des tuteurs (...) Nous avons pu faire le point sur ce qui allait et ce qui n'allait pas. J'ai eu beaucoup de points positifs, c'était satisfaisant » (E6).

31 Seuls deux étudiants sur dix évoquent une diminution de leur sentiment de compétence suite à un feedback jugé négatif: "Les retours que nous avons eus, c'était surtout après la soutenance (...) On nous a remis en question notre manière de fonctionner. Je vois que le sentiment d'auto-efficacité en a pris un petit coup " (E3); "Il y a eu un certain nombre de retours qui ont pointés des négligences, des faiblesses, des résultats qui n'étaient pas à la hauteur des exigences. Cela a conditionné mon cerveau de manière à se dire "attention", à revoir à la baisse ce que j'estimais être capable de faire en terme de gestion de projet » (E8).

De manière générale, les étudiants montrent qu'ils sont sensibles à la perception de leur tuteur (8 sur 10) et de leurs pairs (8 sur 10).: «A un moment donné notre coach [tuteur] m'a dit "Fais, ne réfléchis pas !" (...) Le coach [tuteur] m'a aidée à agir (...) J'ai réussi à me repositionner en tant que partie de l'équipe " (E1); "Le fait d'avoir eu le retour de mes collègues sur cette performance que je donnais, m'a permis de prendre conscience que j'étais finalement impliqué » (E7). Dans une moindre mesure, il ressort que le commanditaire du projet au même titre que les membres du jury de soutenance (professionnels extérieurs, enseignants universitaires, commanditaire du projet) représentent des personnes signifiantes pour quelques étudiants (3 sur 10).

33 En ce qui concerne la manifestation de la persuasion verbale, elle se traduit surtout par des «messages adressés », pour reprendre Galand et Vanlede (2004), qui s'expriment sous diverses formes: «retours» (E1; E3; E5; E6; E8), «avis» (E2; E3; E4; E9), «suggestions» (E7; E8; E9), «feedback» (E5; E8; E9), «encouragements» (E1), « recommandations » (E1), « bonnes critiques » (E2), « commentaires » (E8).

Seulement la moitié des étudiants (5 sur 10) a évoqué les notes obtenues durant leur formation sans forcément établir de relation nette avec leur sentiment de compétence. Trois étudiants sur dix (E1; E3; E8) ont déclaré ne pas avoir accordé d'importance aux notes tandis qu'une minorité ( 2 sur 10) a suggéré que la note pouvait être un indicateur de leur niveau de maîtrise : "Si nous recevons la note qu'à la fin, c'est plus difficile de savoir où nous en sommes" (E6); "C'était aussi intéressant d'avoir plusieurs notes, puisque nous étions notés par plusieurs pairs (...) Si quatre personnes me mettent 10 sur 20 c'est qu'il y a un problème. C'était intéressant de voir ces différents retours 》 (E7).

En dépit du nombre important de notes attribuées dans le cadre de cet enseignement, il ressort que les étudiants prennent davantage en considération les retours constructifs des personnes signifiantes pour eux. 


\section{Discussion et conclusion}

L'objectif de cette recherche visait à étudier les effets d'un dispositif pédagogique sur le sentiment de compétence en gestion de projet des étudiants inscrits en deuxième année de master SEF. Il s'agissait en particulier d'analyser les incidences des modalités pédagogiques ayant recours à la persuasion verbale d'une part et offrant des opportunités d'expériences actives de maîtrise d'autre part. Fondé sur une démarche SoTL, ce travail avait aussi vocation à contribuer à l'amélioration d'une pratique d'enseignement en vue de favoriser le développement professionnel des membres de l'équipe pédagogique.

\subsection{Retours sur les résultats}

Premièrement, les résultats montrent que le sentiment de compétence en gestion de projet des étudiants ayant participé à la formation augmente de manière significative au cours du temps. Autrement dit, plus les étudiants avancent dans leur formation, plus ils se sentent compétents dans le domaine de la gestion de projet, notamment en matière de gestion managériale et de gestion technique. En ce qui concerne la gestion des moyens, aucune amélioration significative n'a été observée entre T2 et T3, c'est-àdire, entre le moment où les étudiants ont terminé le Mooc et celui où ils ont accompli leur mission de gestion de projet dans le cadre du PIC.

Deuxièmement, les propos des étudiants indiquent que les modalités pédagogiques mises en œuvre dans le dispositif Mooc-PIC ont contribué au développement de leur sentiment de compétence en gestion de projet. D'abord, en ce qui concerne l'expérience active de maîtrise, qui représente la source d'information la plus évoquée, il semblerait que les exercices pratiques et les mises en situation réelles, qui étaient proposées aux étudiants, aient favorisé l'évolution observée entre le début et la fin de la formation. Un tel constat conforte ainsi une connaissance ancienne à la fois expérientielle (pédagogues) et empirique (psychologie du développement) qui, de longue date, a mis en évidence le fait que l'expérience active de maîtrise est reconnue comme étant la source d'information la plus importante du sentiment d'efficacité (Bandura, 2003). Ensuite, bien que les étudiants aient moins fait référence à la persuasion verbale, celleci produit le plus souvent un effet positif. Les retours des pairs et du tuteur semblent avoir influencé positivement la perception des étudiants de leurs capacités à gérer un projet, confortant les analyses d'autres auteurs (Lecomte, 2004; Galand, 2011): les retours qui valorisent les points forts et les améliorations, favoriseraient davantage le développement du sentiment de compétence des étudiants que les retours sous forme de note. Troisièmement, les résultats suggèrent que les expériences vicariantes ont également influencé le processus d'autoévaluation dans lequel se sont engagés les étudiants soulignant ainsi l'importance de considérer les différentes sources d'information dans l'analyse du sentiment de compétence. Cependant, cette source reste peu mentionnée au regard des deux autres. Une explication possible est que les étudiants ont eu peu d'occasion, dans le dispositif Mooc-PIC, de s'observer mutuellement dans des situations similaires.

Toutefois, les résultats obtenus ne permettent pas de confirmer l'hypothèse de départ selon laquelle les modalités pédagogiques ayant recours à la persuasion verbale auraient une incidence plus marquée que celles centrées sur l'expérience active de 
maîtrise. Il n'a en effet pas été pas possible d'identifier, à partir de la simple fréquence des propos, la source d'information la plus déterminante du sentiment de compétence en gestion de projet chez les étudiants. Il aurait été sans doute été plus judicieux de recourir à un protocole quasi-expérimental pour connaître de façon précise les effets de chacune des modalités pédagogiques. Par ailleurs, la faiblesse de l'effectif incite à la prudence en vue de toute généralisation.

40 En dépit de ces limites, ce travail présente plusieurs intérêts en particulier pour l'amélioration des pratiques d'enseignement qui était l'un des objectifs initiaux. Il a ainsi permis de dégager plusieurs pistes d'action. D'abord, il est apparu essentiel de réfléchir aux conditions propices à un feedback constructif. Les recherches dans ce domaine (Carless et al., 2011; Boud et Molloy, 2013; Ajjawi et Boud, 2018) montrent qu'il ne suffit pas d'informer les étudiants sur leurs points forts ou leurs points d'amélioration et qu'il convient mieux de les inciter à donner du sens aux informations reçues de sources variées (ex. pairs, enseignants, amis, etc.) (Carless et Boud, 2018). Selon ces auteurs, le feedback doit être appréhendé comme un processus et un dialogue dans lesquels les étudiants jouent un rôle actif. Dans l'expérience présentée ici, il serait donc intéressant d'organiser des feedback par les pairs pour compléter ceux déjà fournis par les commanditaires et les tuteurs. Des temps d'échange pourraient aussi être mis en place pour donner l'occasion aux étudiants de discuter, en toute confiance, des feedback qui leur ont été adressés et ce dans le but de mieux les comprendre et de les utiliser. Ensuite, il est prévu de demander aux étudiants de rédiger un journal réflexif pour les aider à prendre conscience de l'évolution de leurs acquis en termes de compétences. Il s'agira, en particulier : 1) d'exprimer les émotions ressenties, les présupposés de départ et les prises de conscience réalisées tout au long de la formation; 2) d'analyser les expériences vécues (échecs et succès) afin d'identifier les progrès accomplis et de déterminer les actions à entreprendre pour s'améliorer.

41 Le bilan de cette expérience s'est avéré, en outre, très positif du point de vue des étudiants qui en ont souligné les bénéfices. Les éléments d'appréciation concernent la structuration des activités d'apprentissage, le système d'évaluation, le soutien par les pairs et le tuteur ainsi que l'autonomie accordée par le dispositif. Quant aux tuteurs, ils ont tous observé que les étudiants s'étaient fortement impliqués dans la conduite du projet commandité et qu'ils avaient fait preuve d'écoute à leur égard mais aussi entre eux. Ces résultats encourageants invitent à poursuivre les ajustements nécessaires pour permettre aux prochains étudiants de tirer pleinement profit de cette expérimentation.

\subsection{Retours sur la démarche SoTL}

Il convient de reconnaître, au terme de cette analyse, que la démarche SoTL, n'allait pas de soi. Faire de la recherche sur les pratiques d'enseignement en vue de les améliorer a paru, au départ, une entreprise risquée. Si l'intérêt du SoTL pour le développement de l'expertise pédagogique est souvent mis en évidence (Bélanger, 2010; Bédard, 2014; Bélisle et al., 2016), la question de sa légitimité scientifique reste en débat, notamment en France (Rege Colet et Fanghanel, 2015). L'une des craintes initiales était de ne pas pouvoir concilier les trois finalités en jeu dans le SoTL : l'optimisation de la pratique (amélioration de l'enseignement et de l'apprentissage); la transformation de soi (changement de conception, d'approche, de représentations); l'intelligibilité de l'action pédagogique (compréhension du phénomène étudié). La nécessité en sciences de l'éducation et de la formation d'« ouvrir la voie d'une recherche couplée à l'action " 
(Albero, 2014, p. 15), en particulier dans l'enseignement supérieur, a conduit à explorer la démarche SoTL, souvent assimilée à une forme de recherche-action ou encore de recherche appliquée.

Plusieurs intérêts sont apparus en cours d'expérimentation dont la plupart ont déjà été soulignés dans la littérature (Rege Colet et Fanghanel, 2015; Bélisle et al., 2016). Adopter une posture réflexive a permis de clarifier nos intentions pédagogiques et de repérer le manque de cohérence de certaines de nos stratégies d'enseignement (ex. méthodes d'évaluation peu adaptées aux objectifs d'apprentissage). Les investigations réalisées ont conduit à un changement de point de vue porté sur les étudiants, avec la prise de conscience d'habitudes, de techniques, de gestes, voire de croyances pédagogiques pour les questionner en vue de réaliser les ajustements indispensables. Les échanges avec le conseiller pédagogique constituent une autre source d'intérêt de l'approche SoTL. Sa position d'accompagnement, son attitude d'écoute, son travail de médiation aident à gérer les tensions liées à la triple posture d'observateur-acteur-expert inhérente au SoTL. Une démarche collaborative s'est imposée en deux temps pour éviter toute dispersion entre les différents niveaux d'objectifs liés à chacune de ces postures : 1) le temps de l'intervention propre à la recherche de solutions et à l'ingénierie éducative; 2) le temps de la compréhension orientée par le souci de contribuer à la production de connaissances en pédagogie dans l'enseignement supérieur. Pour avoir expérimenté ce processus, nous pouvons considérer le SoTL comme un nouveau type de recherche en éducation qui tend à concilier des visées a priori disjointes (intelligibilité, efficacité, développement personnel/professionnel). Si telle est l'ambition de cette approche, il ne faudrait néanmoins pas tomber dans le piège qui consiste à " réduire l'apprentissage à ce qui est mesurable, l'expertise enseignante à son efficacité, conçue comme valeur ajoutée, et la valeur de l'éducation à son instrumentalité » (Lessard, 2006, p. 30).

\section{BIBLIOGRAPHY}

AFITEP (2000). Dictionnaire de management de projet ( 4 éd.). AFNOR.

Ajjawi, R. et Boud, D. (2018). Examining the nature and effects of feedback dialogue. Assessment \& Evaluation in Higher Education, 43(7), 1106-1119. http://doi.org/10.1080/02602938.2018.1434128

Albero, B. (2014). La pédagogie à l'université entre numérisation et massification. Apports et risques d'une mutation. Dans G. Lameul et C. Loisy (dir.), La pédagogie universitaire à l'heure du numérique: Questionnement et éclairage de la recherche (p. 25-53). De Boeck Supérieur.

Bandura, A. (2003). Auto-efficacité. Le sentiment d'efficacité personnelle (trad. J. Lecomte; $1^{\mathrm{e}}$ éd.). De Boeck Supérieur.

Bédard, D. (2014). Être enseignant ou devenir enseignant dans le supérieur : telle est la question... de posture ! Dans G. Lameul et C. Loisy (dir.), La pédagogie universitaire à l'heure du numérique: Questionnement et éclairage de la recherche (p. 97-109). De Boeck Supérieur. 
Bélanger, C. (2010). Une perspective SoTL au développement professionnel des enseignants au supérieur : Qu'est-ce que cela signifie pour le conseil pédagogique? The Canadian Journal for the Scholarship of Teaching and Learning, 1(2). http://doi.org/10.5206/cjsotl-rcacea.2010.2.6

Bélisle, M., Lison, C. et Bédard, D. (2016). Accompagner le Scholarship of Teaching and Learning. Dans A. Daele et E. Sylvestre (dir.), Comment développer le conseil pédagogique dans l'enseignement supérieur? (p. 75-90). De Boeck Supérieur.

Berger, J.-L. et Büchel, F. (2012). Métacognition et croyances motivationnelles : un mariage de raison. Revue française de pédagogie, 179, 95-128.

Bernstein, D. (2010). Finding Your Place in the Scholarship of Teaching and Learning. International Journal for the Scholarship of Teaching and Learning, 4(2). http://doi.org/10.20429/ ijsotl.2010.040204

Biémar, S., Daele, A., Malengrez, D. et Oger, L. (2015). Le "Scholarship of Teaching and Learning" (SoTL). Proposition d'un cadre pour l'accompagnement des enseignants par les conseillers pédagogiques, Revue internationale de pédagogie de l'enseignement supérieur, 31(2).

Boyer, E. (1990). Scholarship reconsidered: Priorities of the professoriate. The Carnegie Foundation for the Advancement of Teaching.

Boud, D. et Molloy, E. (2013). Rethinking models of feedback for learning : the challenge of design. Assessment \& Evaluation in Higher Education, 38(6), 698-712. http://doi.org/ 10.1080/02602938.2012.691462

Boutin, G. (2004). L'approche par compétences en éducation : un amalgame paradigmatique. Connexions, 1(1), 25-41.

Carless, D. et Boud, D. (2018). The development of student feedback literacy: enabling uptake of feedback. Assessment \& Evaluation in Higher Education, 43(8), 1315-1325. http://doi.org/ 10.1080/02602938.2018.1463354

Carless, D., Salter, D., Yang, M. et Lam, J. (2011). Developing sustainable feedback practices. Studies in Higher Education, 36(4), 395-407. http://doi.org/10.1080/03075071003642449

Carré, P. (2004). Bandura : une psychologie pour le XXI ${ }^{\mathrm{e}}$ siècle. Savoirs, 5, 9-50.

Carré, P. (2010). L'autodirection des apprentissages. Dans D. Poisson, A. Moisan et P. Carré (dir.), L'autoformation, perspectives de recherches (p. 117-169). Presses Universitaires de France.

Cosnefroy, L. (2007). Le sentiment de compétence, un déterminant essentiel de l'intérêt pour les disciplines scolaires. L'orientation scolaire et professionnelle, 36(3), 357-378. https:// journals.openedition.org/osp/1459

Cosnefroy, L. (2010). L'apprentissage autorégulé : perspectives en formation d'adultes. Savoirs, 2(23), 9-50. http://doi.org/10.3917/savo.023.0009

De Ketele, J.-M, Hugonnier, B., Parmentier, P. et Cosnefroy, L. (2016). Quelle excellence pour l'enseignement supérieur? De Boeck Supérieur.

Endrizzi, L. (2011). Savoir enseigner dans le supérieur : un enjeu d'excellence pédagogique. Dossier d'actualité Veille \& Analyses, 64. http://veille-et-analyses.ens-lyon.fr/DA-Veille/64septembre-2011.pdf

Galand, B. (2011). Avoir confiance en soi. Dans E. Bourgeois et G. Chapelle (dir.), Apprendre et faire apprendre (p. 255-268). Presses Universitaires de France. 
Galand, B. et Vanlede, M. (2004). Le sentiment d'efficacité personnelle dans l'apprentissage et la formation : quel rôle joue-t-il? D’où vient-il? Comment intervenir? Savoirs, 5, 91-116.

Gerard, F.-M., Huggonier, B. et Varin, S. (2018, janvier). Mesure de la qualité des systèmes éducatifs des pays de l'OCDE [communication]. 30 colloque de l'ADME-Europe, Esch-sur-Alzette, Luxembourg. http://www.fmgerard.be/textes/mesure_qualite.html

Granier, C., Mas, L., Finot, L., Arnoux, B., Pasqualini, N. et Dollé, V. (2009). La démarche qualité dans la recherche publique et l'enseignement supérieur. Éditions Quæ.

Guy, S. et Huber, E. (2014). Améliorer le sentiment d'efficacité personnelle par l'enseignement explicite : analyse de deux situations d'élèves à besoins éducatifs particuliers en intégration scolaire [mémoire de master]. Haute école pédagogique du canton de Vaud. http://doc.rero.ch/record/259246/files/ md_maes_p17107_p22025_2014.pdf

Jacob, L. (2018). Les classes inversées en premier cycle universitaire : de la motivation initiale à l'autorégulation de l'apprentissage [thèse de doctorat inédite]. Université de Strasbourg.

Lanarès, J. et Poteaux, N. (2013). Comment répondre aux défis actuels de l'enseignement supérieur? Dans D. Berthiaume et N. Rege Colet (dir.), La pédagogie de l'enseignement supérieur : repères théoriques et applications pratiques. Tome 1 : Enseigner au supérieur (p. 9-24). Peter Lang.

Langevin, L. (dir.). (2007). Formation et soutien à l'enseignement universitaire : des constats et des exemples pour inspirer l'action. Presses de l'Université du Québec.

Langevin, L., Grandtner, A.-M. et Ménard, L. (2008). La formation à l'enseignement des professeurs d'université : Un aperçu. Revue des sciences de l'éducation, 34(3), 643-664. http:// doi.org/10.7202/029512ar

Lecomte, J. (2004). Les applications du sentiment d'efficacité personnelle. Savoirs, 5, 59-90. Lessard, C. (2006). Le débat américain sur la certification des enseignants et le piège d'une politique éducative evidence-based. Revue française de pédagogie, 154, 19-32. http://doi.org/ $10.4000 / \mathrm{rfp} .108$

Lison, C. (2013). La pratique réflexive en enseignement supérieur : d'une approche théorique à une perspective de développement professionnel. Phronesis, 2(1), 15-27. http://doi.org/ 10.7202/1015636ar

Melançon, J., Lefebvre, S. et Thibodeau, S. (2013). Sources d'influence de l'auto-efficacité relative à un enseignement intégrant les TIC chez des enseignants du primaire. Éducation et Francophonie, 21(1). http://doi.org/10.7202/1015060ar

Miles, M. B. et Huberman, A. M. (2003). Analyse des données qualitatives (trad. M. Hlady Rispal; $2^{\mathrm{e}}$ éd.). De Boeck Supérieur.

Muchielli, R. (2006). L'analyse de contenu des documents et des communications (9e éd.). ESF éditeur.

Noël, B. et Cartier, S. (dir.). (2016). De la métacognition à l'apprentissage autorégulé. De Boeck Supérieur.

Peguret, M. (2014). Le sentiment d'autoefficacité et les stratégies d'apprentissage chez les étudiants de français langue seconde venant d'immersion. Revue des sciences de l'éducation, 40(3), 579-599. http://doi.org/10.7202/1029075ar

Rege Colet, N. et Fanghanel, J. (2015). Faire de la recherche appliquée sur ses enseignements. Dans N. Rege Colet et D. Berthiaume (dir.), La pédagogie de l'enseignement supérieur : repères théoriques et applications pratiques. Tome 2 : Se développer au titre d'enseignant (p. 221-234). Peter Lang. 
Rege Colet, N., McAlpine, L., Fanghanel, J. et Weston, C. (2011). Le concept de Scholarship of Teaching and Learning. Recherche \& Formation, 67, 91-104. https://journals.openedition.org/ rechercheformation/1412

Rege Colet, N. et Romainville, M. (dir.). (2006). La pratique enseignante en mutation à l'université. De Boeck Supérieur.

Roegiers, X. (2014). Quelle évaluation des compétences, au service de quel projet pour l'école? Dans C. Dierendonck (dir.), L'évaluation des compétences en milieu scolaire et en milieu professionnel (p. 71-83). De Boeck Supérieur.

Schön, D. A. (1983). Le praticien réflexif : À la recherche du savoir caché dans l'agir professionnel. Éditions logiques.

Zimmerman, B. J. (1995). Self-efficacy and educational development. Dans A. Bandura, (dir.), Selfefficacy in Changing Societies (p. 202-231). Cambridge University Press.

Zimmerman, B. J. (2000). Self-Efficacy: An Essential Motive to Learn. Contemporary Educational Psychology, 25, 82-91. http://doi.org/10.1006/ceps.1999.1016

\section{NOTES}

1. Les plans stratégiques de 5 universités ont été consultés à partir d'une recherche sur Google. Ces établissements étaient classés dans les 100 premiers rangs du Palmarès de Shanghai 2020 : University of California Berkeley Strategic Plan 2018-2020 ; The University of British Columbia's Strategic Plan 2018-2028; Plan stratégique de l'Université de Genève - Une vision pour 2025 ; National University of Singapore - Annual Report 2019 ; Projet stratégique de l'Université Grenoble Alpes 2016-2020. Dans un souci de lisibilité, les liens de téléchargement ne sont pas indiqués.

2. Le questionnaire comprenait 9 items liés à la " gestion managériale » relative au champ des ressources humaines et à la communication (ex. impliquer les membres de l'équipe en valorisant leur travail, animer une réunion en suivant l'ordre du jour, etc.), 7 items concernant la " gestion technique " liée aux équipements et aux outils de suivi (ex. utiliser un outil d'organisation collaboratif, rédiger un rapport d'étape, etc.) et 8 items relatifs à la " gestion des moyens " propre à la planification et au suivi budgétaire (ex. estimer le coût d'un projet, établir un calendrier prévisionnel, etc.).

3. 1) « je ne suis absolument pas en mesure de réaliser la tâche même avec un accompagnement ";2) « je peux réaliser la tâche si je bénéficie d'un accompagnement »;3) «j'ai une bonne maîtrise de la tâche et je suis en mesure de la réaliser de manière autonome »;4) "j'ai une excellente maîtrise de la tâche et je peux aussi former un collègue à la faire ».

4. La faiblesse de l'effectif et la non-normalité des distributions nous a conduit à utiliser le test de Wilcoxon, qui est une alternative non paramétrique au test $t$ de Student, pour des échantillons appariés. 


\section{ABSTRACTS}

This paper presents the results of a scholarship of teaching and learning study, which has been conducted in order to improve project management instruction for students enrolled in the second year of the Master of Educational Sciences at the University of Strasbourg. After we noticed that students didn't feel ready for leading a project at the end of their program, we have developed a new pedagogical approach based on the Self-Efficacy Theory (Bandura, 2003). The aim of the SoTL work, initiated at the same time, was to examine whether the introduction of the verbal persuasion instructional model had a more positive effect on the project management self-competency than the learning-by-doing approach used previously. So, students have been invited to assess themselves on the 24 competencies targeted by this course, at three moments : before, during and after their program experience. Semi-structured interviews were also conducted with all the participants. While results show a significant increase in project management self-competency, it has been impossible to establish a strong relationship with either of both pedagogical approach. However, the results of this experience were very positive and some strategies for practice have been suggested for the future. This contribution ends with a reflection on our own experience of the SoTL approach.

Cet article présente les résultats d'une recherche réalisée dans une perspective Scholarship of Teaching and Learning (SoTL) visant à améliorer un enseignement de gestion de projet proposé en deuxième année de master de sciences de l'éducation et de la formation à l'Université de Strasbourg. Après avoir observé que les étudiants ne se sentaient pas prêts à conduire un projet à l'issue de leur formation, une nouvelle formule pédagogique a été mise en place, étayée par la théorie du sentiment d'auto-efficacité (Bandura, 2003). La démarche SoTL, conduite dans le même temps, devait permettre de vérifier si l'introduction de modalités pédagogiques ayant recours à la persuasion verbale avait une incidence positive plus marquée sur le sentiment de compétence en gestion de projet que les situations d'expériences actives de maîtrise déjà mises en œuvre antérieurement. Pour ce faire, les étudiants $(\mathrm{n}=10)$ ont été invités à s'autoévaluer sur les 24 compétences visées par cet enseignement et ce, à trois reprises : avant, pendant et après leur participation à la formation. Des entretiens semi-directifs ont également été réalisés avec tous les participants. Si les résultats montrent une augmentation significative du sentiment de compétence en gestion de projet, il n'a pas été possible d'établir de liens précis avec l'une ou l'autre des méthodes pédagogiques mises en place. Cependant, le bilan de cette expérimentation s'est avéré positif et des pistes d'action ont pu être envisagées pour la suite. Cette contribution se termine par une réflexion sur la mobilisation de l'approche SoTL dans ce contexte.

\section{INDEX}

Mots-clés: Scholarship of teaching and learning, sentiment de compétence, sources de l'efficacité personnelle, gestion de projet 


\section{AUTHORS}

\section{NAJOUA MOHIB}

Université de Strasbourg, Université de Haute-Alsace, Université de Lorraine, Laboratoire Interuniversitaire de Sciences de l'Éducation et de la Communication (LISEC - UR 2310), 7, Strasbourg, France, najoua.mohib@unistra.fr

\section{SIMON ZINGARETTI}

Université de Strasbourg, Institut de développement et d'innovation pédagogiques, Strasbourg, France, zingaretti@unistra.fr

\section{RÉMI BACHELET}

Centrale Lille, PRES Université Lille Nord de France, Villeneuve d'Ascq, remi.bachelet@centralelille.fr 